Punjab University Journal of Mathematics (2021),53(8),575-592

https://doi.org/10.52280/pujm.2021.530804

\title{
Petrović's type inequality for exponentially convex functions and coordinated
} exponentially convex functions

\author{
Wasim Iqbal \\ COMSATS University Islamabad, Islamabad, Pakistan. \\ Email: waseem.iqbal.attock@gmail.com \\ Muhammad Aslam Noor \\ COMSATS University Islamabad, Islamabad, Pakistan. \\ email: noormaslam@gmail.com \\ Khalida Inayat Noor \\ COMSATS University Islamabad, Islamabad, Pakistan. \\ email: khalidan@gmail.com
}

Received: 21 February, 2020 / Accepted: 12 July, 2021 / Published online: 26 August, 2021

\begin{abstract}
In this paper, we produce a novel framework of a subclass of convex functions that is exponentially convex functions. Moreover, it is observed that the new concept helps to build new inequalities of Petrović's type by employing exponentially convex functions. We also introduce the idea of coordinated exponentially convex functions and derive Petrović's type inequality for coordinated exponentially convex functions. We also find Lagrange type and Cauchy type mean value theorems for Petrović's type inequality for exponentially convex and coordinated exponentially convex functions. Our consequences with this new generalizations has the abilities to be implemented for the evaluation of many mathematical problems.
\end{abstract}

AMS (MOS) Subject Classification Codes: 26A51; 26D15

Key Words: Petrović's inequality, exponentially convex functions, exponentially convex functions on coordinates.

\section{INTRODUCTION}

Integral inequalities are marvelous tools for building up the qualitative and quantitative properties of convex functions. There has been a ceaseless development of intrigue in such a region of research so as to address the issues of different utilizations of these variants. For example, inequalities have been contemplated by numerous analysts who thus utilized assorted procedures for investigating and proposing these variants [12, 18, 29]. One of the 
most significant inequality is the distinguished Petrović's inequality [22]. Petrović's type inequality have been obtained by several authors, see [8, 21, 22, 23, 24, 25, 26, 27, 28, 10] and reference therein.

Another approach is efficient to obtain the integral inequalities by utilizing convex functions. It is known that the subclass of convex functions is closely related to log-convex functions referred to as exponentially convex functions. Exponentially convex function explored by Bernstein [7] in covariance formation then Avriel [4] contemplated and investigated this concept by imposing the condition of $r$-convex functions. Noor et. al. [13] explored exponentially convex functions while studying the paper of Antczak [3]. For more features concerning to exponentially convex functions, see $[2,5,13,15,17]$ and the references therein. Pal [20] provided the fertile application of exponentially convex functions in information theory, optimization theory, and statistical theory. For observing various other kinds of exponentially convex functions and their generalizations, see [14, 16, 20].

The main purpose of this paper is to introduce a new concept of exponentially convex functions on coordinates. We derive Petrović's type inequality for exponentially convex and coordinated exponentially convex functions. We expect that the idea may open new doors in futuristic research.

\section{Notations AND PREliminaries}

In this section, we demonstrate the basic definitions concerning to our research.

Definition 1. A nonempty set $\Omega \subseteq \mathbb{R}$ is convex, if

$$
\sigma \eta+(1-\sigma) \xi \in \Omega, \quad \forall \eta, \xi \in \Omega, \quad \sigma \in[0,1] .
$$

Definition 2. A function $\mathcal{F}: \Omega \rightarrow \mathbb{R}$ is convex, if

$$
\mathcal{F}(\sigma \eta+(1-\sigma) \xi) \leq \sigma \mathcal{F}(\eta)+(1-\sigma) \mathcal{F}(\xi), \quad \forall \eta, \xi \in \Omega, \quad \sigma \in[0,1] .
$$

Definition 3.([13]) A positive function $\mathcal{F}$ is called exponentially convex function on $\Omega$, if

$$
e^{\mathcal{F}(\eta+\sigma(\xi-\eta))} \leq(1-\sigma) e^{\mathcal{F}(\eta)}+\sigma e^{\mathcal{F}(\xi)}, \quad \forall \eta, \xi \in \Omega, \quad \sigma \in[0,1],
$$

which can be written in the following form, which is due to Avriel [4].

Definition 4. A positive function $\mathcal{F}$ is called exponentially convex function on $\Omega$, if

$$
\mathcal{F}(\eta+\sigma(\xi-\eta)) \leq \log \left[(1-\sigma) e^{\mathcal{F}(\eta)}+\sigma e^{\mathcal{F}(\xi)}\right], \quad \forall \eta, \xi \in \Omega, \quad \sigma \in[0,1] .
$$

For the applications of the exponentially convex functions in information theory and mathematical programming, see Antczak [3] and Alirezaei and Mathar [2]. 
From now onwards, we take $I_{1}=\left[a_{1}, b_{1}\right]$ and $I_{2}=\left[c_{1}, d_{1}\right]$ as intervals in $\mathbb{R}$.

Other aspects of exponentially convex functions can be expressed as:

$$
e^{\mathcal{F}(\eta)} \leq \frac{\eta-b_{1}}{a_{1}-b_{1}} e^{\mathcal{F}\left(a_{1}\right)}+\frac{\eta-a_{1}}{b_{1}-a_{1}} e^{\mathcal{F}\left(b_{1}\right)}, \quad \forall \eta \in I=\left[0, b_{1}\right],
$$

equivalently, one can write

$$
\frac{e^{\mathcal{F}(\eta)}-e^{\mathcal{F}\left(a_{1}\right)}}{\eta-a_{1}} \leq \frac{e^{\mathcal{F}\left(b_{1}\right)}-e^{\mathcal{F}\left(a_{1}\right)}}{b_{1}-a_{1}}, \quad \forall \eta \in I,
$$

which shows $\mathcal{F}$ is increasing in $I$.

Dragomir [9] introduced coordinated convex functions as follows:

Definition 5.([9]) Suppose the bidimensional interval $\Delta=I_{1} \times I_{2}$. Also, let $\mathcal{F}: \Delta \rightarrow \mathbb{R}$ be a mapping. Define partial mappings as

$$
\mathcal{F}_{\xi}:\left[a_{1}, b_{1}\right] \rightarrow \mathbb{R} \text { defined by } \mathcal{F}_{\xi}\left(u_{1}\right)=\mathcal{F}\left(u_{1}, \xi\right)
$$

and

$$
\mathcal{F}_{\eta}:\left[c_{1}, d_{1}\right] \rightarrow \mathbb{R} \text { defined by } \mathcal{F}_{\eta}\left(v_{1}\right)=\mathcal{F}\left(\eta, v_{1}\right) .
$$

The function $\mathcal{F}$ is called coordinated convex, if the partial mappings defined in (2. 4 ) and (2. 5$)$ are convex on $\left[a_{1}, b_{1}\right]$ and $\left[c_{1}, d_{1}\right]$ respectively, for all $\xi \in\left[c_{1}, d_{1}\right]$ and $\eta \in$ $\left[a_{1}, b_{1}\right]$.

Definition 6. The function $\mathcal{F}: \Delta \rightarrow \mathbb{R}$ is convex in $\Delta$, if

$$
\begin{aligned}
\mathcal{F}\left(\sigma \eta+(1-\sigma) z_{1}, \sigma \xi+(1-\sigma) w_{1}\right) & \leq \sigma \mathcal{F}(\eta, \xi)+(1-\sigma) \mathcal{F}\left(z_{1}, w_{1}\right), \\
& \forall(\eta, \xi),\left(z_{1}, w_{1}\right) \in \Delta, \sigma \in[0,1] .
\end{aligned}
$$

We now define the coordinated exponentially convex functions.

Definition 7. Suppose the bidimensional interval $\Delta_{1}=\left[0, b_{1}\right] \times\left[0, d_{1}\right]$. Also, let $\mathcal{F}: \Delta_{1} \rightarrow \mathbb{R}$ be a positive mapping. Define partial mappings as

$$
\mathcal{F}_{\xi}:\left[0, b_{1}\right] \rightarrow \mathbb{R} \text { defined by } \mathcal{F}_{\xi}\left(u_{1}\right)=\mathcal{F}\left(u_{1}, \xi\right)
$$

and

$$
\mathcal{F}_{\eta}:\left[0, d_{1}\right] \rightarrow \mathbb{R} \text { defined by } \mathcal{F}_{\eta}\left(v_{1}\right)=\mathcal{F}\left(\eta, v_{1}\right) \text {. }
$$

The function $\mathcal{F}$ is coordinated exponentially convex, if the partial mappings defined in $(2.7)$ and $(2.8)$ are exponentially convex on $\left[0, b_{1}\right]$ and $\left[0, d_{1}\right]$ respectively, for all $\xi \in\left[0, d_{1}\right]$ and $\eta \in\left[0, b_{1}\right]$.

Definition 8. Let $\Delta_{1} \subseteq \mathbb{R}^{n}$. A positive mapping $\mathcal{F}: \Delta_{1} \rightarrow \mathbb{R}$ is exponentially convex in $\Delta_{1}$, if

$$
\begin{array}{r}
e^{\mathcal{F}\left(\sigma \eta+(1-\sigma) z_{1}, \sigma \xi+(1-\sigma) w_{1}\right)} \leq \sigma e^{\mathcal{F}(\eta, \xi)}+(1-\sigma) e^{\mathcal{F}\left(z_{1}, w_{1}\right)}, \\
\forall(\eta, \xi),\left(z_{1}, w_{1}\right) \in \Delta_{1}, \sigma \in[0,1] .
\end{array}
$$

Lemma 2.1. Every exponentially convex mapping $\mathcal{F}: \Delta_{1} \rightarrow \mathbb{R}$ is coordinated exponentially convex, but converse is not true in general. 
Proof. Let a positive mapping $\mathcal{F}: \Delta_{1} \rightarrow \mathbb{R}$ be an exponentially convex in $\Delta_{1}$. Also, let $\mathcal{F}_{\eta}:\left[0, d_{1}\right] \rightarrow \mathbb{R}$ defined as $\mathcal{F}_{\eta}\left(v_{1}\right):=\mathcal{F}\left(\eta, v_{1}\right)$. Then

$$
\begin{aligned}
e^{\mathcal{F}_{\eta}\left(\sigma v_{1}+(1-\sigma) w_{1}\right)} & =e^{\mathcal{F}\left(\eta, \sigma v_{1}+(1-\sigma) w_{1}\right)} \\
& =e^{\mathcal{F}\left(\sigma \eta+(1-\sigma) \eta, \sigma v_{1}+(1-\sigma) w_{1}\right)} \\
& \leq \sigma e^{\mathcal{F}\left(\eta, v_{1}\right)}+(1-\sigma) e^{\mathcal{F}\left(\eta, w_{1}\right)} \\
& =\sigma e^{\mathcal{F}_{\eta}\left(v_{1}\right)}+(1-\sigma) e^{\mathcal{F}_{\eta}\left(w_{1}\right)}, \quad \forall \sigma \in[0,1] \text { and } v_{1}, w_{1} \in\left[0, d_{1}\right],
\end{aligned}
$$

which shows the exponentially convexity of $\mathcal{F}_{\eta}$.

Similarly, one can show the exponentially convexity of $\mathcal{F}_{\xi}$.

Now, consider the positive mapping $\mathcal{F}_{0}:[0,1]^{2} \rightarrow[0, \infty)$ given by $e^{\mathcal{F}_{0}\left(u_{1}, v_{1}\right)}=e^{u_{1} v_{1}}$. Clearly $\mathcal{F}$ is coordinated exponentially convex. But it is not exponentially convex on $[0,1]^{2}$.

Indeed, if $(\eta, 0),\left(0, w_{1}\right) \in[0,1]^{2}$ and $\sigma \in[0,1]$. Then

$$
e^{\mathcal{F}\left(\sigma(\eta, 0)+(1-\sigma)\left(0, w_{1}\right)\right)}=e^{\mathcal{F}\left(\sigma \eta,(1-\sigma) w_{1}\right)}=e^{\sigma(1-\sigma) \eta w_{1}}
$$

and

$$
\sigma e^{\mathcal{F}(\eta, 0)}+(1-\sigma) e^{\mathcal{F}\left(0, w_{1}\right)}=1=e^{0} .
$$

Thus, $\forall \sigma \in(0,1), \eta, w_{1} \in(0,1)$, one has

$$
e^{\mathcal{F}\left(\sigma(\eta, 0)+(1-\sigma)\left(0, w_{1}\right)\right)}>\sigma e^{\mathcal{F}(\eta, 0)}+(1-\sigma) e^{\mathcal{F}\left(0, w_{1}\right)},
$$

which shows that $\mathcal{F}$ is not exponentially convex.

Petrović [22] derived some inequality for convex functions.

Theorem 1.([22]) Let $\left(\eta_{i}, i=1,2, \ldots, n\right)$ be non-negative n-tuples and $\left(p_{j}, j=1,2, \ldots, n\right)$ be positive n-tuples such that $\sum_{j=1}^{n} p_{j} \geq 1$,

$$
\sum_{\kappa=1}^{n} p_{\kappa} \eta_{\kappa} \in\left[0, a_{1}\right] \text { and } \sum_{\kappa=1}^{n} p_{\kappa} \eta_{\kappa} \geq \eta_{l} \text { for each } l=1, \ldots, n \text {. }
$$

Consider the function $\mathcal{F}$ is convex on $\left[0, a_{1}\right]$, then

$$
\sum_{\kappa=1}^{n} p_{\kappa} \mathcal{F}\left(\eta_{\kappa}\right) \leq \mathcal{F}\left(\sum_{\kappa=1}^{n} p_{\kappa} \eta_{\kappa}\right)+\left(\sum_{\kappa=1}^{n} p_{\kappa}-1\right) \mathcal{F}(0)
$$

is valid.

Rehman et al. [28] gave the Petrović's inequality on coordinated convex functions.

Theorem 2. ([28]) Let $\left(\eta_{i}, i=1,2, \ldots, n\right)$ and $\left(\xi_{j}, j=1,2, \ldots, n\right)$ be non-negative $\mathrm{n}$-tuples and $\left(p_{k}, k=1, \ldots, n\right)$ and $\left(q_{l}, l=1, \ldots, n\right)$ be positive $\mathrm{n}$-tuples such that

$$
\sum_{\kappa=1}^{n} p_{\kappa} \geq 1, \quad 0 \neq \sum_{\kappa=1}^{n} p_{\kappa} \eta_{\kappa} \geq \eta_{j} \text { for every } j=1,2, \ldots, n
$$


and

$$
\sum_{l=1}^{n} q_{l} \geq 1, \quad 0 \neq \sum_{l=1}^{n} q_{l} \xi_{l} \geq \xi_{j} \text { for every } i=1,2, \ldots, n .
$$

Let $\mathcal{F}:\left[0, a_{1}\right) \times\left[0, b_{1}\right) \rightarrow \mathbb{R}$ be a convex on coordinates, then

$$
\begin{aligned}
& \sum_{\kappa=1}^{n} \sum_{l=1}^{n} p_{\kappa} q_{l} \mathcal{F}\left(\eta_{\kappa}, \xi_{l}\right) \leqslant \mathcal{F}\left(\sum_{\kappa=1}^{n} p_{\kappa} \eta_{\kappa}, \sum_{l=1}^{n} q_{l} \xi_{l}\right)+\left(\sum_{l=1}^{n} q_{l}-1\right) \mathcal{F}\left(\sum_{\kappa=1}^{n} p_{\kappa} \eta_{\kappa}, 0\right) \\
& +\left(\sum_{\kappa=1}^{n} p_{\kappa}-1\right)\left(\mathcal{F}\left(0, \sum_{l=1}^{n} q_{l} \xi_{l}\right)+\left(\sum_{l=1}^{n} q_{l}-1\right) \mathcal{F}(0,0)\right) .
\end{aligned}
$$

\section{RESUlTS}

In this section, we inagurate a lemma, which plays a key role for proving our next results associated with exponentially convex functions.

Lemma 3.1. Let $\left(\eta_{i}, i=1,2, \ldots, n\right)$ be non-negative $n$-tuples and $\left(p_{j}, j=1,2, \ldots, n\right)$ be positive $n$-tuples such that $\sum_{j=1}^{n} p_{j} \geq 1, \theta \in\left[0, a_{1}\right]$,

$$
\sum_{\kappa=1}^{n} p_{\kappa} \eta_{\kappa} \in\left[0, a_{1}\right] \text { and } \sum_{\kappa=1}^{n} p_{\kappa} \eta_{\kappa} \geq \eta_{l}>\theta \text { for each } l=1, \ldots, n \text {. }
$$

Suppose a positive function $\mathcal{F}:\left[0, a_{1}\right] \rightarrow \mathbb{R}$ is exponentially convex. If $\frac{e^{\mathcal{F}(\eta)}}{\eta-\theta}$ is increasing on $\left[0, a_{1}\right]$, then

$$
e^{\mathcal{F} \sum_{\kappa=1}^{n} p_{\kappa} \eta_{\kappa}} \geq \frac{\left(\sum_{\kappa=1}^{n} p_{\kappa} \eta_{\kappa}-\theta\right)}{\sum_{\kappa=1}^{n} p_{\kappa}\left(\eta_{\kappa}-\theta\right)} \sum_{\kappa=1}^{n} p_{\kappa} e^{\mathcal{F}\left(\eta_{\kappa}\right)}
$$

Proof. Since $\sum_{\kappa=1}^{n} p_{\kappa} \eta_{\kappa} \geq \eta_{l}>\theta$ for all $l=1, \ldots, n$ and $\frac{e^{\mathcal{F}(\eta)}}{\eta-\theta}$ is increasing on $\left[0, a_{1}\right]$, we have

$$
\frac{e^{\mathcal{F} \sum_{\kappa=1}^{n} p_{\kappa} \eta_{\kappa}}}{\left(\sum_{\kappa=1}^{n} p_{\kappa} \eta_{\kappa}-\theta\right)} \geq \frac{e^{\mathcal{F}\left(\eta_{\kappa}\right)}}{\left(\eta_{\kappa}-\theta\right)}
$$

This implies

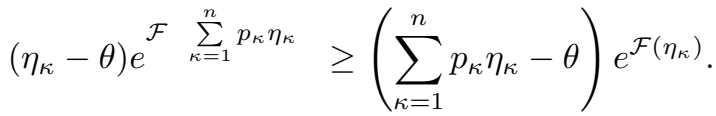

Multiplying above inequality by $p_{\kappa}$ and taking sum for $\kappa=1, \ldots, n$, one has

$$
\sum_{\kappa=1}^{n} p_{\kappa}\left(\eta_{\kappa}-\theta\right) e^{\mathcal{F} \sum_{\kappa=1}^{n} p_{\kappa} \eta_{\kappa}} \geq\left(\sum_{\kappa=1}^{n} p_{\kappa} \eta_{\kappa}-\theta\right) \sum_{l=1}^{n} p_{\kappa} e^{\mathcal{F}\left(\eta_{\kappa}\right)}
$$


from which, one has the required result.

We now derive the Petrovićs type inequality for exponentially convex functions.

Theorem 3. Let $\left(\eta_{i}, i=1,2, \ldots, n\right)$ be non-negative n-tuples and $\left(p_{j}, j=1,2, \ldots, n\right)$ be positive n-tuples such that $\sum_{j=1}^{n} p_{j} \geq 1, \theta \in\left[0, a_{1}\right]$,

$$
\sum_{\kappa=1}^{n} p_{\kappa} \eta_{\kappa} \in\left[0, a_{1}\right] \text { and } \sum_{\kappa=1}^{n} p_{\kappa} \eta_{\kappa} \geq \eta_{l}>\theta \text { for each } l=1, \ldots, n \text {. }
$$

Let a positive function $\mathcal{F}:[0, \infty) \rightarrow \mathbb{R}$ be an exponentially convex and $\frac{e^{\mathcal{F}(\eta)}}{\eta-\theta}$ is increasing on $\left[0, a_{1}\right]$. Then

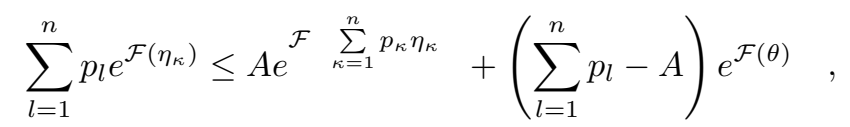

where

$$
A=\left(\frac{\sum_{l=1}^{n} p_{l}\left(\eta_{l}-\theta\right)}{\sum_{\kappa=1}^{n} p_{\kappa} \eta_{\kappa}-\theta}\right)
$$

Proof. Since $\mathcal{F}$ is exponentially convex. so from Lemma 3.1,

$$
e^{\mathcal{F} \sum_{\kappa=1}^{n} p_{\kappa} \eta_{\kappa}}-e^{\mathcal{F}(\theta)} \geq \frac{\left(\sum_{\kappa=1}^{n} p_{\kappa} \eta_{\kappa}-\theta\right)}{\sum_{l=1}^{n} p_{l}\left(\eta_{l}-\theta\right)} \sum_{l=1}^{n} p_{l}\left(e^{\mathcal{F}\left(\eta_{\kappa}\right)}-e^{\mathcal{F}(\theta)}\right) .
$$

This gives us

$$
\frac{\sum_{l=1}^{n} p_{l}\left(\eta_{l}-\theta\right)}{\sum_{\kappa=1}^{n} p_{\kappa} \eta_{\kappa}-\theta}\left(e^{\mathcal{F} \sum_{\kappa=1}^{n} p_{\kappa} \eta_{\kappa}}-e^{\mathcal{F}(\theta)}\right) \geq \sum_{l=1}^{n} p_{l} e^{\mathcal{F}\left(\eta_{\kappa}\right)}-\sum_{l=1}^{n} p_{l} e^{\mathcal{F}(\theta)}
$$

This leads to

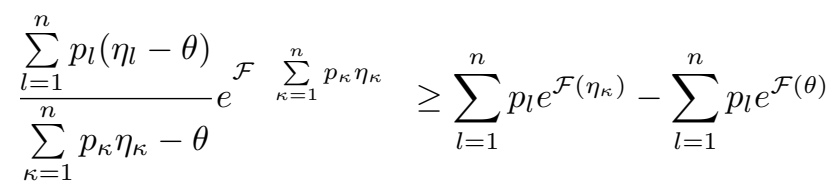

$$
\begin{aligned}
& +\left(\frac{\sum_{l=1}^{n} p_{l}\left(\eta_{l}-\theta\right)}{\sum_{\kappa=1}^{n} p_{\kappa} \eta_{\kappa}-\theta}\right) e^{\mathcal{F}(\theta)}
\end{aligned}
$$


Finally, we have

$$
\begin{aligned}
& \frac{\sum_{l=1}^{n} p_{l}\left(\eta_{l}-\theta\right)}{\sum_{\kappa=1}^{n} p_{\kappa} \eta_{\kappa}-\theta} e^{\mathcal{F}} \geq \sum_{l=1}^{\sum_{\kappa=1}^{n} p_{\kappa} \eta_{\kappa}} p_{l} e^{\mathcal{F}\left(\eta_{\kappa}\right)}- \\
& \left(\sum_{l=1}^{n} p_{l}-\frac{\sum_{l=1}^{n} p_{l}\left(\eta_{l}-\theta\right)}{\sum_{\kappa=1}^{n} p_{\kappa} \eta_{\kappa}-\theta}\right) e^{\mathcal{F}(\theta)} .
\end{aligned}
$$

This is equivalent to the required result.

If $\theta=0$, then Theorem 3 reduces to the following new result.

Theorem 4. Let the conditions given in Theorem 3 be satisfied. Also, let a positive function $\mathcal{F}:[0, \infty) \rightarrow \mathbb{R}$ be an exponentially convex. Then

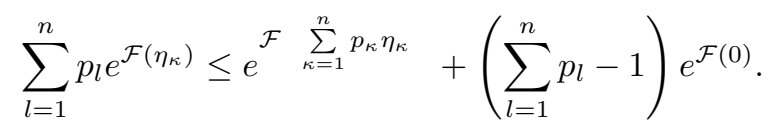

Now, we derive Petrović's type inequality on coordinated exponentially convex functions.

Theorem 5. Let $\left(\eta_{i}, i=1,2, \ldots, n\right)$ and $\left(\xi_{j}, j=1,2, \ldots, n\right)$ be non-negative n-tuples and $\left(p_{k}, k=1,2, \ldots, n\right)$ and $\left(q_{l}, l=1, \ldots, n\right)$ be positive n-tuples such that $\theta \in\left[0, a_{1}\right]$, $\sum_{\kappa=1}^{n} p_{\kappa} \geq 1, \sum_{l=1}^{n} q_{l} \geq 1$,

$$
\sum_{\kappa=1}^{n} p_{\kappa} \eta_{\kappa} \in\left[0, a_{1}\right), \quad 0 \neq \sum_{\kappa=1}^{n} p_{\kappa} \eta_{\kappa} \geq \eta_{j}>\theta \text { for every } j=1,2, \ldots, n
$$

and

$$
\sum_{l=1}^{n} q_{l} \xi_{l} \in\left[0, b_{1}\right), \quad 0 \neq \sum_{l=1}^{n} q_{l} \xi_{l} \geq \xi_{i}>\theta \text { for every } i=1,2, \ldots, n .
$$

Let a positive function $\mathcal{F}:[0, \infty)^{2} \rightarrow \mathbb{R}$ be coordinated exponentially convex function and $\frac{e^{\mathcal{F}(\eta)}}{\eta-\theta}$ is increasing on $\left[0, a_{1}\right]$. Then

$$
\begin{aligned}
& \sum_{\kappa=1}^{n} \sum_{l=1}^{n} p_{\kappa} q_{l} e^{\mathcal{F}\left(\eta_{j}, \xi_{l}\right)} \leq A\left\{B e^{\mathcal{F} \sum_{\kappa=1}^{n} p_{\kappa} \eta_{\kappa}, \sum_{l=1}^{n} q_{l} \xi_{l}}+\left(\sum_{l=1}^{n} q_{l}-B\right) e^{\mathcal{F} \sum_{\kappa=1}^{n} p_{\kappa} \eta_{\kappa}, \theta}\right\} \\
& +\left(\sum_{\kappa=1}^{n} p_{\kappa}-A\right)\left\{B e^{\mathcal{F} \theta, \sum_{l=1}^{n} q_{l} \xi_{l}}+\left(\sum_{l=1}^{n} q_{l}-B\right) e^{\mathcal{F}(\theta, \theta)}\right\}
\end{aligned}
$$

where 


$$
A=\left(\frac{\sum_{\kappa=1}^{n} p_{\kappa}\left(\eta_{\kappa}-\theta\right)}{\sum_{\kappa=1}^{n} p_{\kappa} \eta_{\kappa}-\theta}\right)
$$

and

$$
B=\left(\frac{\sum_{l=1}^{n} q_{l}\left(\xi_{l}-\theta\right)}{\sum_{l=1}^{n} q_{l} \xi_{l}-\theta}\right) .
$$

Proof. Consider the partial mappings $\mathcal{F}_{\eta}:\left[0, a_{1}\right] \rightarrow \mathbb{R}$ and $\mathcal{F}_{\xi}:\left[0, b_{1}\right] \rightarrow \mathbb{R}$ defined by $\mathcal{F}_{\eta}\left(v_{1}\right)=\mathcal{F}\left(\eta, v_{1}\right)$ and $\mathcal{F}_{\xi}\left(u_{1}\right)=\mathcal{F}\left(u_{1}, \xi\right)$.

As $\mathcal{F}$ is coordinated exponentially convex on $[0, \infty)^{2}$. Therefore, the partial mapping $\mathcal{F}_{\xi}$ is exponentially convex on $\left[0, b_{1}\right]$. By Theorem 3 , we have

$$
\begin{aligned}
\sum_{\kappa=1}^{n} p_{\kappa} e^{\mathcal{F}_{\xi}\left(\eta_{j}\right)} & \leq\left(\frac{\sum_{\kappa=1}^{n} p_{\kappa}\left(\eta_{\kappa}-\theta\right)}{\sum_{\kappa=1}^{n} p_{\kappa} \eta_{\kappa}-\theta}\right) e^{\mathcal{F}_{\xi}} \sum_{\kappa=1}^{n} p_{\kappa} \eta_{\kappa} \\
& +\left(\sum_{\kappa=1}^{n} p_{\kappa}-\frac{\sum_{\kappa=1}^{n} p_{\kappa}\left(\eta_{\kappa}-\theta\right)}{\sum_{\kappa=1}^{n} p_{\kappa} \eta_{\kappa}-\theta}\right) e^{\mathcal{F}_{\xi}(\theta)}
\end{aligned}
$$

This is equivalent to

$$
\begin{aligned}
& \sum_{\kappa=1}^{n} p_{\kappa} e^{\mathcal{F}\left(\eta_{j}, \xi\right)} \leq\left(\frac{\sum_{\kappa=1}^{n} p_{\kappa}\left(\eta_{\kappa}-\theta\right)}{\sum_{\kappa=1}^{n} p_{\kappa} \eta_{\kappa}-\theta}\right) e^{\mathcal{F}} \sum_{\kappa=1}^{n} p_{\kappa} \eta_{\kappa}, \xi \\
&+\left(\sum_{\kappa=1}^{n} p_{\kappa}-\frac{\sum_{\kappa=1}^{n} p_{\kappa}\left(\eta_{\kappa}-\theta\right)}{\sum_{\kappa=1}^{n} p_{\kappa} \eta_{\kappa}-\theta}\right) e^{\mathcal{F}(\theta, \xi)} .
\end{aligned}
$$


By setting $\xi=\xi_{l}$, we get

$$
\begin{aligned}
\sum_{\kappa=1}^{n} p_{\kappa} e^{\mathcal{F}\left(\eta_{j}, \xi_{l}\right)} & \leq\left(\frac{\sum_{\kappa=1}^{n} p_{\kappa}\left(\eta_{\kappa}-\theta\right)}{\sum_{\kappa=1}^{n} p_{\kappa} \eta_{\kappa}-\theta}\right) e^{\mathcal{F} \sum_{\kappa=1}^{n} p_{\kappa} \eta_{\kappa}, \xi_{l}} \\
& +\left(\sum_{\kappa=1}^{n} p_{\kappa}-\frac{\sum_{\kappa=1}^{n} p_{\kappa}\left(\eta_{\kappa}-\theta\right)}{\sum_{\kappa=1}^{n} p_{\kappa} \eta_{\kappa}-\theta}\right) e^{\mathcal{F}\left(\theta, \xi_{l}\right)}
\end{aligned}
$$

Multiplying above inequality by $q_{l}$ and taking sum for $l=1, \ldots, n$, one has

$$
\begin{aligned}
& \sum_{\kappa=1}^{n} \sum_{l=1}^{n} p_{\kappa} q_{l} e^{\mathcal{F}\left(\eta_{j}, \xi_{l}\right)} \leq\left(\frac{\sum_{\kappa=1}^{n} p_{\kappa}\left(\eta_{\kappa}-\theta\right)}{\sum_{\kappa=1}^{n} p_{\kappa} \eta_{\kappa}-\theta}\right) \sum_{l=1}^{n} q_{l} e^{\mathcal{F} \sum_{\kappa=1}^{n} p_{\kappa} \eta_{\kappa}, \xi_{l}}+ \\
& \left(\sum_{\kappa=1}^{n} p_{\kappa}-\frac{\sum_{\kappa=1}^{n} p_{\kappa}\left(\eta_{\kappa}-\theta\right)}{\sum_{\kappa=1}^{n} p_{\kappa} \eta_{\kappa}-\theta}\right) \sum_{l=1}^{n} q_{l} e^{\mathcal{F}\left(\theta, \xi_{l}\right)} .
\end{aligned}
$$

Now again by Theorem 3, we have

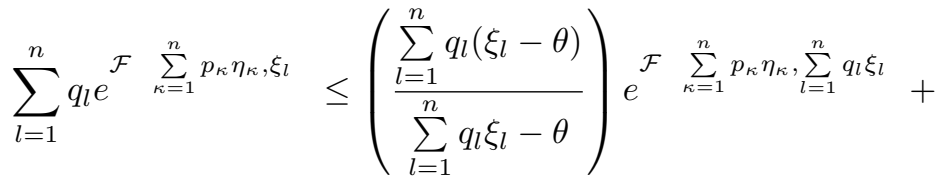

$$
\begin{aligned}
& \left(\sum_{l=1}^{n} q_{l}-\frac{\sum_{l=1}^{n} q_{l}\left(\xi_{l}-\theta\right)}{\sum_{l=1}^{n} q_{l} \xi_{l}-\theta}\right) e^{\mathcal{F}} \sum_{\kappa=1}^{n} p_{\kappa} \eta_{\kappa}, \theta
\end{aligned}
$$

and

$$
\begin{aligned}
\sum_{l=1}^{n} q_{l} e^{\mathcal{F}\left(\theta, \xi_{l}\right)} & \leq\left(\frac{\sum_{l=1}^{n} q_{l}\left(\xi_{l}-\theta\right)}{\sum_{l=1}^{n} q_{l} \xi_{l}-\theta}\right) e^{\mathcal{F} \theta, \sum_{l=1}^{n} q_{l} \xi_{l}} \\
+ & \left(\sum_{l=1}^{n} q_{l}-\frac{\sum_{l=1}^{n} q_{l}\left(\xi_{l}-\theta\right)}{\sum_{l=1}^{n} q_{l} \xi_{l}-\theta}\right) e^{\mathcal{F}(\theta, \theta)} .
\end{aligned}
$$

Putting these values in inequality (3.18) and using the notations given in (3.16) and (3. 17$)$, we get the required result.

If $\theta=0$, then Theorem 3 reduces to the following new result. 
Theorem 6. Let the conditions given in Theorem 3 be satisfied. If $\mathcal{F}:[0, \infty)^{2} \rightarrow \mathbb{R}$ be coordinated exponentially convex, then

$$
\begin{aligned}
& \sum_{\kappa=1}^{n} \sum_{l=1}^{n} p_{\kappa} q_{l} e^{\mathcal{F}\left(\eta_{j}, \xi_{l}\right)} \leq\left\{e^{\mathcal{F} \sum_{\kappa=1}^{n} p_{\kappa} \eta_{\kappa}, \sum_{l=1}^{n} q_{l} \xi_{l}}+\left(\sum_{l=1}^{n} q_{l}-1\right) e^{\mathcal{F} \sum_{\kappa=1}^{n} p_{\kappa} \eta_{\kappa}, 0}\right\} \\
& +\left(\sum_{\kappa=1}^{n} p_{\kappa}-1\right)\left\{e^{\mathcal{F} 0, \sum_{l=1}^{n} q_{l} \xi_{l}}+\left(\sum_{l=1}^{n} q_{l}-1\right) e^{\mathcal{F}(0,0)}\right\} .
\end{aligned}
$$

By considering non-negative difference of (3.14), we define the following linear functional.

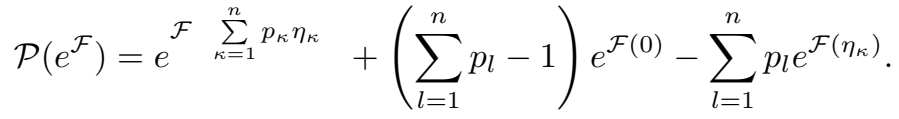

Also by considering non-negative difference of (3.19), we define the following linear functional.

$$
\begin{aligned}
& \Upsilon\left(e^{\mathcal{F}}\right)=\left\{e^{\mathcal{F} \sum_{\kappa=1}^{n} p_{\kappa} \eta_{\kappa}, \sum_{l=1}^{n} q_{l} \xi_{l}}+\left(\sum_{l=1}^{n} q_{l}-1\right) e^{\mathcal{F} \sum_{\kappa=1}^{n} p_{\kappa} \eta_{\kappa}, 0}\right\} \\
& +\left(\sum_{\kappa=1}^{n} p_{\kappa}-1\right)\left\{e^{\mathcal{F} 0, \sum_{l=1}^{n} q_{l} \xi_{l}}+\left(\sum_{l=1}^{n} q_{l}-1\right) e^{\mathcal{F}(0,0)}\right\}-\sum_{\kappa=1}^{n} \sum_{l=1}^{n} p_{\kappa} q_{l} e^{\mathcal{F}\left(\eta_{j}, \xi_{l}\right)} .
\end{aligned}
$$

We need the following lemma.

Lemma 3.2. Let a positive function $\mathcal{F}:\left[0, b_{1}\right] \rightarrow \mathbb{R}$ be an exponentially convex such that

$$
n_{1} \leqslant \frac{\left(\eta-a_{1}\right) e^{\mathcal{F}(\eta)} \mathcal{F}^{\prime}(\eta)-e^{\mathcal{F}(\eta)}+e^{\mathcal{F}\left(a_{1}\right)}}{\left(\eta-a_{1}\right)^{2}} \leqslant N_{1}
$$

$\forall \eta \in\left[0, b_{1}\right] \backslash\left\{a_{1}\right\}$ and $a_{1} \in\left(0, b_{1}\right)$.

Let $\gamma_{1}, \gamma_{2}:\left[0, b_{1}\right] \rightarrow \mathbb{R}$ be positive functions defined as

$$
\gamma_{1}(\eta)=\log \left[N_{1} \eta^{2}-e^{\mathcal{F}(\eta)}\right]
$$

and

$$
\gamma_{2}(\eta)=\log \left[e^{\mathcal{F}(\eta)}-n_{1} \eta^{2}\right]
$$

then $\gamma_{1}$ and $\gamma_{2}$ are exponentially convex on $\left[0, b_{1}\right]$.

Proof. Suppose

$$
P_{\gamma_{1}}(\eta)=\frac{e^{\gamma_{1}(\eta)}-e^{\gamma_{1}\left(a_{1}\right)}}{\eta-a_{1}}
$$




$$
\begin{aligned}
& =\frac{N_{1} \eta^{2}-e^{\mathcal{F}(\eta)}-N_{1} a_{1}^{2}+e^{\mathcal{F}\left(a_{1}\right)}}{\eta-a_{1}} \\
& =\frac{N_{1}\left(\eta^{2}-a_{1}^{2}\right)}{\eta-a_{1}}-\frac{e^{\mathcal{F}(\eta)}-e^{\mathcal{F}\left(a_{1}\right)}}{\eta-a_{1}} . \\
& =N_{1}\left(\eta+a_{1}\right)-\frac{e^{\mathcal{F}(\eta)}-e^{\mathcal{F}\left(a_{1}\right)}}{\eta-a_{1}} .
\end{aligned}
$$

By differentiating with respect to $\eta$, one has

$$
P_{\gamma_{1}}^{\prime}(\eta)=N_{1}-\frac{\left(\eta-a_{1}\right) e^{\mathcal{F}(\eta)} \mathcal{F}^{\prime}(\eta)-e^{\mathcal{F}(\eta)}+e^{\mathcal{F}\left(a_{1}\right)}}{\left(\eta-a_{1}\right)^{2}} .
$$

Since

This implies

$$
N_{1}-\frac{\left(\eta-a_{1}\right) e^{\mathcal{F}(\eta)} \mathcal{F}^{\prime}(\eta)-e^{\mathcal{F}(\eta)}+e^{\mathcal{F}\left(a_{1}\right)}}{\left(\eta-a_{1}\right)^{2}} \geq 0
$$

$$
P_{\gamma_{1}}^{\prime}(\eta) \geq 0, \forall \eta \in\left[0, a_{1}\right) \cup\left(a_{1}, b_{1}\right] .
$$

Similarly, one can show that

$$
P_{\gamma_{2}}^{\prime}(\eta) \geq 0, \quad \forall \eta \in\left[0, a_{1}\right) \cup\left(a_{1}, b_{1}\right] .
$$

This implies that $P_{\gamma_{1}}$ and $P_{\gamma_{2}}$ are increasing on $\eta \in\left[0, a_{1}\right) \cup\left(a_{1}, b_{1}\right]$ for all $a \in\left(0, b_{1}\right)$. Hence by $(2.3), \gamma_{1}(\eta)$ and $\gamma_{2}(\eta)$ are exponentially convex in $\left[0, b_{1}\right]$.

Here we give mean value theorems related to functional defined for Petrović's inequality for exponentially convex functions.

Theorem 7. Let $\left(\eta_{1}, \ldots, \eta_{n}\right) \in\left[0, b_{1}\right]$, and $\left(p_{1}, \ldots, p_{n}\right)$ be positive n-tuples such that $\sum_{k=1}^{n} p_{k} \eta_{k} \geq \eta_{j}$ for each $j=1,2, \ldots, n$.

Also let $\phi(\eta)=\log \eta^{2}$.

If a positive exponentially convex function $\mathcal{F} \in C^{1}\left(\left[0, b_{1}\right]\right)$, then there exist $\eta \in\left(0, b_{1}\right)$ such that

$$
\mathcal{P}\left(e^{\mathcal{F}}\right)=\frac{(\eta-a) e^{\mathcal{F}(\eta)} \mathcal{F}^{\prime}(\eta)-e^{\mathcal{F}(\eta)}+e^{\mathcal{F}\left(a_{1}\right)}}{(\eta-a)^{2}} \mathcal{P}\left(e^{\phi}\right),
$$

provided that $\mathcal{P}\left(e^{\phi}\right)$ is non zero and $a \in\left(0, b_{1}\right)$, where $\mathcal{P}\left(e^{\mathcal{F}}\right)$ is a linear functional.

Proof. As $\mathcal{F} \in C^{1}\left(\left[0, b_{1}\right]\right)$, so there exist real numbers $n_{1}$ and $N_{1}$ such that

$$
n_{1} \leqslant \frac{\left(\eta-a_{1}\right) e^{\mathcal{F}(\eta)} \mathcal{F}^{\prime}(\eta)-e^{\mathcal{F}(\eta)}+e^{\mathcal{F}\left(a_{1}\right)}}{\left(\eta-a_{1}\right)^{2}} \leqslant N_{1}, \quad \forall \eta \in\left[0, b_{1}\right] \text { and } a_{1} \in\left(0, b_{1}\right) \text {. }
$$

Consider the functions $\gamma_{1}$ and $\gamma_{2}$ defined in Lemma 3.2.

As $\gamma_{1}$ is exponentially convex in $\left[0, b_{1}\right]$, so

$$
\mathcal{P}\left(e^{\gamma_{1}}\right) \geq 0
$$


that is

this gives

$$
\mathcal{P}\left(N_{1} \eta^{2}-e^{\mathcal{F}(\eta)}\right) \geq 0
$$

$$
N_{1} \mathcal{P}\left(e^{\phi}\right) \geq \mathcal{P}\left(e^{\mathcal{F}}\right) .
$$

Similarly $\gamma_{2}$ is exponentially convex $\left[0, b_{1}\right]$, therefore one has

$$
n_{1} \mathcal{P}(\phi) \leqslant \mathcal{P}\left(e^{\mathcal{F}}\right) \text {. }
$$

By assumption $\mathcal{P}\left(e^{\phi}\right)$ is non zero, combining inequalities (3. 23 ) and (3. 24), one has

$$
n_{1} \leqslant \frac{\mathcal{P}\left(e^{\mathcal{F}}\right)}{\mathcal{P}\left(e^{\phi}\right)} \leqslant N_{1} .
$$

Hence there exist $\eta \in\left(0, b_{1}\right)$ such that

$$
\frac{\mathcal{P}\left(e^{\mathcal{F}}\right)}{\mathcal{P}\left(e^{\phi}\right)}=\frac{(\eta-a) e^{\mathcal{F}(\eta)} \mathcal{F}^{\prime}(\eta)-e^{\mathcal{F}(\eta)}+e^{\mathcal{F}\left(a_{1}\right)}}{(\eta-a)^{2}},
$$

which is the required result.

Theorem 8. Let the conditions given in Theorem 3 be satisfied.

Suppose the positive exponentially convex functions $\mathcal{F}_{1}, \mathcal{F}_{2} \in C^{1}\left(\left[0, b_{1}\right]\right)$, then there exist $\xi \in\left(0, b_{1}\right)$ such that

$$
\frac{\mathcal{P}\left(e^{\mathcal{F}_{1}}\right)}{\mathcal{P}\left(e^{\mathcal{F}_{2}}\right)}=\frac{(\xi-a) e^{\mathcal{F}_{1}(\xi)} \mathcal{F}_{1}^{\prime}(\xi)-e^{\mathcal{F}_{1}(\xi)}+e^{\mathcal{F}_{1}(a)}}{(\xi-a) e^{\mathcal{F}_{2}(\xi)} \mathcal{F}_{2}^{\prime}(\xi)-e^{\mathcal{F}_{2}(\xi)}+e^{\mathcal{F}_{2}(a)}}
$$

provided that the denominators are non-zero and $a_{1} \in\left(0, b_{1}\right)$, where $\mathcal{P}\left(e^{\mathcal{F}_{1}}\right)$ and $\mathcal{P}\left(e^{\mathcal{F}_{2}}\right)$ are linear functional.

Proof. Suppose $k \in C^{1}\left(\left[0, b_{1}\right]\right)$ be a function defined as

$$
k=\log \left(c_{1} e^{\mathcal{F}_{1}}-c_{2} e^{\mathcal{F}_{2}}\right)
$$

where $c_{1}$ and $c_{2}$ are defined as

$$
\begin{aligned}
& c_{1}=\mathcal{P}\left(e^{\mathcal{F}_{2}}\right), \\
& c_{2}=\mathcal{P}\left(e^{\mathcal{F}_{1}}\right) .
\end{aligned}
$$

Then using Theorem 3 with $\mathcal{F}=k$, one has

$$
\begin{aligned}
& (\xi-a) e^{\log \left(c_{1} e^{\mathcal{F}_{1}(\xi)}-c_{2} e^{\mathcal{F}_{2}(\xi)}\right)}\left(\log \left(c_{1} e^{\mathcal{F}_{1}(\xi)}-c_{2} e^{\mathcal{F}_{2}(\xi)}\right)\right)^{\prime}-\left(c_{1} e^{\mathcal{F}_{1}(\xi)}-c_{2} e^{\mathcal{F}_{2}(\xi)}\right) \\
& +\left(c_{1} e^{\mathcal{F}_{1}(a)}-c_{2} e^{\mathcal{F}_{2}(a)}\right)=0
\end{aligned}
$$

this gives

$$
(\xi-a)\left(c_{1} e^{\mathcal{F}_{1}(\xi)}-c_{2} e^{\mathcal{F}_{2}(\xi)}\right)^{\prime}-c_{1} e^{\mathcal{F}_{1}(\xi)}+c_{2} e^{\mathcal{F}_{2}(\xi)}+c_{1} e^{\mathcal{F}_{1}(a)}-c_{2} e^{\mathcal{F}_{2}(a)}=0
$$

that is

$(\xi-a)\left(c_{1} e^{\mathcal{F}_{1}(\xi)} \mathcal{F}_{1}^{\prime}(\xi)-c_{2} e^{\mathcal{F}_{2}(\xi)} \mathcal{F}_{2}^{\prime}(\xi)\right)-c_{1} e^{\mathcal{F}_{1}(\xi)}+c_{2} e^{\mathcal{F}_{2}(\xi)}+c_{1} e^{\mathcal{F}_{1}(a)}-c_{2} e^{\mathcal{F}_{2}(a)}=0$, this gives

$(\xi-a) c_{1} e^{\mathcal{F}_{1}(\xi)} \mathcal{F}_{1}^{\prime}(\xi)-(\xi-a) c_{2} e^{\mathcal{F}_{2}(\xi)} \mathcal{F}_{2}^{\prime}(\xi)-c_{1} e^{\mathcal{F}_{1}(\xi)}+c_{2} e^{\mathcal{F}_{2}(\xi)}+c_{1} e^{\mathcal{F}_{1}(a)}-c_{2} e^{\mathcal{F}_{2}(a)}=0$ 
this implies

$$
\begin{gathered}
c_{1}\left\{(\xi-a) e^{\mathcal{F}_{1}(\xi)} \mathcal{F}_{1}^{\prime}(\xi)-e^{\mathcal{F}_{1}(\xi)}+e^{\mathcal{F}_{1}(a)}\right\}=c_{2}\left\{(\xi-a) e^{\mathcal{F}_{2}(\xi)} \mathcal{F}_{2}^{\prime}(\xi)-e^{\mathcal{F}_{2}(\xi)}+e^{\mathcal{F}_{2}(a)}\right\} \\
\frac{c_{2}}{c_{1}}=\frac{(\xi-a) e^{\mathcal{F}_{1}(\xi)} \mathcal{F}_{1}^{\prime}(\xi)-e^{\mathcal{F}_{1}(\xi)}+e^{\mathcal{F}_{1}(a)}}{(\xi-a) e^{\mathcal{F}_{2}(\xi)} \mathcal{F}_{2}^{\prime}(\xi)-e^{\mathcal{F}_{2}(\xi)}+e^{\mathcal{F}_{2}(a)}}
\end{gathered}
$$

Putting the values of $c_{1}$ and $c_{2}$, one has the required result.

We need the following lemma.

Lemma 3.3. Let $\Delta=\left[0, b_{1}\right] \times\left[0, d_{1}\right]$. Also, let $\mathcal{F}: \Delta \rightarrow \mathbb{R}$ be a positive coordinated exponentially convex function such that

$$
n_{1} \leqslant \frac{\left(\eta-a_{1}\right) e^{\mathcal{F}(\eta, \xi)} \frac{\partial}{\partial \eta} \mathcal{F}(\eta, \xi)-e^{\mathcal{F}(\eta, \xi)}+e^{\mathcal{F}\left(a_{1}, \xi\right)}}{\left(\eta-a_{1}\right)^{2} \xi^{2}} \leqslant N_{1}
$$

and

$$
n_{2} \leqslant \frac{\left(\xi-c_{1}\right) e^{\mathcal{F}(\eta, \xi)} \frac{\partial}{\partial \xi} \mathcal{F}(\eta, \xi)-e^{\mathcal{F}(\eta, \xi)}+e^{\mathcal{F}\left(\eta, c_{1}\right)}}{\left(\xi-c_{1}\right)^{2} \eta^{2}} \leqslant N_{2}
$$

$\forall \eta \in\left[0, b_{1}\right] \backslash\left\{a_{1}\right\}, a_{1} \in\left(0, b_{1}\right)$ and $\xi \in\left[0, d_{1}\right] \backslash\left\{c_{1}\right\}, c \in\left(0, d_{1}\right)$.

Consider the functions $\alpha_{\xi}:\left[0, b_{1}\right] \rightarrow \mathbb{R}$, and $\alpha_{\eta}:\left[0, d_{1}\right] \rightarrow \mathbb{R}$, defined as

$$
\alpha(\eta, \xi)=\log \left[\max \left\{N_{1}, N_{2}\right\} \eta^{2} \xi^{2}-e^{\mathcal{F}(\eta, \xi)}\right]
$$

and

$$
\beta(\eta, \xi)=\log \left[e^{\mathcal{F}(\eta, \xi)}-\min \left\{n_{1}, n_{2}\right\} \eta^{2} \xi^{2}\right] .
$$

Then $\alpha$ and $\beta$ are coordinated exponentially convex.

Proof. Suppose the partial mappings $\alpha_{\xi}:\left[0, b_{1}\right] \rightarrow \mathbb{R}$ and $\alpha_{\eta}:\left[0, d_{1}\right] \rightarrow \mathbb{R}$ defined as $\alpha_{\xi}(\eta):=\alpha(\eta, \xi)$ for all $\eta \in\left(0, b_{1}\right]$ and $\alpha_{\eta}(\xi):=\alpha(\eta, \xi)$ for all $\xi \in\left(0, d_{1}\right]$.

$$
\begin{aligned}
P_{\alpha_{\xi}}(\eta) & =\frac{e^{\alpha_{\xi}(\eta)}-e^{\alpha_{\xi}\left(a_{1}\right)}}{\eta-a_{1}} \\
& =\frac{e^{\alpha(\eta, \xi)}-e^{\alpha\left(a_{1}, \xi\right)}}{\eta-a_{1}} \\
& =\frac{e^{\log \left[\max \left\{N_{1}, N_{2}\right\} \eta^{2} \xi^{2}-e^{\mathcal{F}(\eta, \xi)}\right]}-e^{\log \left[\max \left\{N_{1}, N_{2}\right\} a^{2} \xi^{2}-e^{\mathcal{F}\left(a_{1}, \xi\right)}\right]}}{\eta-a_{1}} \\
& =\frac{N_{1} \eta^{2} \xi^{2}-e^{\mathcal{F}(\eta, \xi)}-N_{1} a^{2} \xi^{2}+e^{\mathcal{F}\left(a_{1}, \xi\right)}}{\eta-a_{1}} \\
& =N_{1} \frac{\left(\eta^{2}-a^{2}\right) \xi^{2}}{\eta-a_{1}}-\frac{e^{\mathcal{F}(\eta, \xi)}-e^{\mathcal{F}\left(a_{1}, \xi\right)}}{\eta-a_{1}} \\
& =N_{1}(\eta+a) \xi^{2}-\frac{e^{\mathcal{F}(\eta, \xi)}-e^{\mathcal{F}\left(a_{1}, \xi\right)}}{\eta-a_{1}}
\end{aligned}
$$


Differentiating partially with respect to $\eta$, one has

$$
\begin{aligned}
P_{\alpha_{\xi}}^{\prime}(\eta) & =N_{1} \frac{\partial}{\partial \eta}\left(\eta \xi^{2}+a_{1} \xi^{2}\right)-\frac{\partial}{\partial \eta}\left(\frac{e^{\mathcal{F}(\eta, \xi)}-e^{\mathcal{F}\left(a_{1}, \xi\right)}}{\eta-a_{1}}\right) \\
& =N_{1} \xi^{2}-\frac{\left(\eta-a_{1}\right) e^{\mathcal{F}(\eta, \xi)} \frac{\partial}{\partial \eta} \mathcal{F}(\eta, \xi)-e^{\mathcal{F}(\eta, \xi)}+e^{\mathcal{F}\left(a_{1}, \xi\right)}}{\left(\eta-a_{1}\right)^{2}} .
\end{aligned}
$$

By assumption

$$
\begin{gathered}
N_{1} \xi^{2}-\frac{\left(\eta-a_{1}\right) e^{\mathcal{F}(\eta, \xi)} \frac{\partial}{\partial \eta} \mathcal{F}(\eta, \xi)-e^{\mathcal{F}(\eta, \xi)}+e^{\mathcal{F}\left(a_{1}, \xi\right)}}{\left(\eta-a_{1}\right)^{2}} \geq 0 \\
P_{\alpha_{\xi}}^{\prime}(\eta) \geq 0, \quad \forall \eta \in\left[0, a_{1}\right) \cup\left(a_{1}, b_{1}\right]
\end{gathered}
$$

Similarly, one can show that

$$
P_{\alpha_{\eta}}^{\prime}(\xi) \geq 0, \forall \eta \in\left[0, c_{1}\right) \cup\left(c_{1}, d_{1}\right] .
$$

This ensure that $P_{\alpha_{\xi}}$ is increasing on $\left[0, a_{1}\right) \cup\left(a_{1}, b_{1}\right]$ for all $a_{1} \in\left[0, b_{1}\right]$ and $P_{\alpha_{\eta}}$ is increasing on $\left[0, c_{1}\right) \cup\left(c_{1}, d_{1}\right]$ for all $c_{1} \in\left[0, d_{1}\right]$.

By (2. 3 ), $\alpha$ is exponentially convex. Hence by Lemma $2.1, \alpha$ is coordinated exponentially convex.

Similarly, one can show that $\beta$ is coordinated exponentially convex.

Here we give mean value theorems related to the functional defined for Petrovic̀'s inequality for coordinated exponentially convex functions.

Theorem 9. Let $\left(\eta_{1}, \ldots, \eta_{n}\right) \in\left[0, b_{1}\right],\left(\xi_{1}, \ldots, \xi_{n}\right) \in\left[0, d_{1}\right]$ be non-negative $n$-tuples and $\left(q_{1}, \ldots, q_{n}\right),\left(p_{1}, \ldots, p_{n}\right)$ be positive n-tuples such that

$\sum_{k=1}^{n} p_{k} \eta_{k} \geq \eta_{j}$ for each $j=1,2, \ldots, n$. Also let $\varphi(\eta, \xi)=\log \left(\eta^{2} \xi^{2}\right)$.

Let a positive coordinated exponentially convex function $\mathcal{F} \in C^{1}(\Delta)$, then there exist $(\xi, \eta)$ in the interior of $\Delta$, such that

$$
\Upsilon\left(e^{\mathcal{F}}\right)=\frac{(\eta-a) e^{\mathcal{F}(\xi, \eta)} \frac{\partial}{\partial \eta} \mathcal{F}(\xi, \eta)-e^{\mathcal{F}(\xi, \eta)}+e^{\mathcal{F}(\xi, a)}}{(\eta-a)^{2} \xi^{2}} \Upsilon\left(e^{\varphi}\right)
$$

and

$$
\Upsilon\left(e^{\mathcal{F}}\right)=\frac{(\xi-a) e^{\mathcal{F}(\xi, \eta)} \frac{\partial}{\partial \xi} \mathcal{F}(\xi, \eta)-e^{\mathcal{F}(\xi, \eta)}+e^{\mathcal{F}(a, \eta)}}{(\xi-a)^{2} \eta^{2}} \Upsilon\left(e^{\varphi}\right),
$$

provided that $\Upsilon\left(e^{\varphi}\right)$ is non-zero and $a \in\left(0, b_{1}\right)$, where $\Upsilon\left(e^{\mathcal{F}}\right)$ is a linear functional.

Proof. As $\mathcal{F}$ has continuous first order partial derivative in $\Delta$, so there exist real numbers $n_{1}, n_{2}, N_{1}$ and $N_{2}$ such that

$$
n_{1} \leqslant \frac{(\eta-a) e^{\mathcal{F}(\eta, \xi)} \frac{\partial}{\partial \eta} \mathcal{F}(\eta, \xi)-e^{\mathcal{F}(\eta, \xi)}+e^{\mathcal{F}(\xi, a)}}{(\eta-a)^{2} \xi^{2}} \leqslant N_{1}
$$


and

$$
n_{2} \leqslant \frac{(\xi-a) e^{\mathcal{F}(\eta, \xi)} \frac{\partial}{\partial \xi} \mathcal{F}(\eta, \xi)-e^{\mathcal{F}(\eta, \xi)}+e^{\mathcal{F}(a, \eta)}}{(\xi-a)^{2} \eta^{2}} \leqslant N_{2},
$$

$\forall \eta \in\left(0, b_{1}\right], \xi \in(0, d]$ and $a \in\left(0, b_{1}\right)$.

Consider the functions $\alpha$ and $\beta$ defined in Lemma 3.3.

As $\alpha$ is coordinated exponentially convex, then

$$
\Upsilon\left(e^{\alpha}\right) \geq 0
$$

that is

$$
\Upsilon\left(N_{1} \eta^{2} \xi^{2}-e^{\mathcal{F}(\eta, \xi)}\right) \geq 0
$$

this gives

$$
N_{1} \Upsilon\left(e^{\varphi}\right) \geq \Upsilon\left(e^{\mathcal{F}}\right)
$$

Similarly $\beta$ is coordinated exponentially convex, therefore one has

$$
n_{1} \Upsilon\left(e^{\varphi}\right) \leqslant \Upsilon\left(e^{\mathcal{F}}\right)
$$

By assumption $\Upsilon\left(e^{\varphi}\right)$ is non-zero, so combining inequalities (3. 27 ) and (3. 28 ), one has

$$
n_{1} \leqslant \frac{\Upsilon\left(e^{\mathcal{F}}\right)}{\Upsilon\left(e^{\varphi}\right)} \leqslant N_{1}
$$

Hence there exists $(\xi, \eta)$ in the interior of $\Delta$, such that

$$
\Upsilon\left(e^{\mathcal{F}}\right)=\frac{(\eta-a) e^{\mathcal{F}(\xi, \eta)} \frac{\partial}{\partial \eta} \mathcal{F}(\xi, \eta)-e^{\mathcal{F}(\xi, \eta)}+e^{\mathcal{F}(\xi, a)}}{(\eta-a)^{2} \eta^{2}} \Upsilon\left(e^{\varphi}\right) .
$$

Similarly, one can show that

$$
\Upsilon\left(e^{\mathcal{F}}\right)=\frac{(\xi-a) e^{\mathcal{F}(\xi, \eta)} \frac{\partial}{\partial \xi} \mathcal{F}(\xi, \eta)-e^{\mathcal{F}(\xi, \eta)}+e^{\mathcal{F}(a, \eta)}}{(\xi-a)^{2} \eta^{2}} \Upsilon\left(e^{\varphi}\right),
$$

which is the required result.

Theorem 10. Let the conditions given in Theorem 3 be satisfied.

Also let the positive coordinated exponentially convex functions $\mathcal{F}_{1}, \mathcal{F}_{2} \in C^{1}(\Delta)$, then there exist $(\xi, \eta)$ in the interior of $\Delta$, such that

$$
\frac{\Upsilon\left(e^{\mathcal{F}_{1}}\right)}{\Upsilon\left(e^{\mathcal{F}_{2}}\right)}=\frac{(\eta-a) e^{\mathcal{F}_{1}(\xi, \eta)} \frac{\partial}{\partial \eta} \mathcal{F}_{1}(\xi, \eta)-e^{\mathcal{F}_{1}(\xi, \eta)}+e^{\mathcal{F}_{1}(\xi, a)}}{(\eta-a) e^{\mathcal{F}_{2}(\xi, \eta)} \frac{\partial}{\partial \eta} \mathcal{F}_{2}(\xi, \eta)-e^{\mathcal{F}_{2}(\xi, \eta)}+e^{\mathcal{F}_{2}(\xi, a)}}
$$

and

$$
\frac{\Upsilon\left(e^{\mathcal{F}_{1}}\right)}{\Upsilon\left(e^{\mathcal{F}_{2}}\right)}=\frac{(\xi-a) e^{\mathcal{F}_{1}(\xi, \eta)} \frac{\partial}{\partial \xi} \mathcal{F}_{1}(\xi, \eta)-e^{\mathcal{F}_{1}(\xi, \eta)}+e^{\mathcal{F}_{1}(a, \eta)}}{(\xi-a) e^{\mathcal{F}_{2}(\xi, \eta)} \frac{\partial}{\partial \xi} \mathcal{F}_{2}(\xi, \eta)-e^{\mathcal{F}_{2}(\xi, \eta)}+e^{\mathcal{F}_{2}(a, \eta)}},
$$

provided that the denominators are non-zero and $a \in\left(0, b_{1}\right)$, where $\Upsilon\left(e^{\mathcal{F}_{1}}\right)$ and $\Upsilon\left(e^{\mathcal{F}_{2}}\right)$ are linear functional. 
Proof. Suppose

$$
k=\log \left(c_{1} e^{\mathcal{F}_{1}}-c_{2} e^{\mathcal{F}_{2}}\right)
$$

where $c_{1}$ and $c_{2}$ are defined as

$$
\begin{aligned}
c_{1} & =\Upsilon\left(e^{\mathcal{F}_{2}}\right), \\
c_{2} & =\Upsilon\left(e^{\mathcal{F}_{1}}\right) .
\end{aligned}
$$

Using Theorem 3 with $\mathcal{F}=k$, one has

$$
\begin{aligned}
& (\eta-a) e^{\log \left(c_{1} e^{\mathcal{F}_{1}}-c_{2} e^{\mathcal{F}_{2}}\right)(\xi, \eta)} \frac{\partial}{\partial \eta} \log \left(c_{1} e^{\mathcal{F}_{1}}-c_{2} e^{\mathcal{F}_{2}}\right)(\xi, \eta)-e^{\log \left(c_{1} e^{\mathcal{F}_{1}}-c_{2} e^{\mathcal{F}_{2}}\right)(\xi, \eta)} \\
& +e^{\log \left(c_{1} e^{\mathcal{F}_{1}}-c_{2} e^{\mathcal{F}_{2}}\right)(\xi, a)}=0 \\
& (\eta-a) \frac{\partial}{\partial \eta}\left(c_{1} e^{\mathcal{F}_{1}}-c_{2} e^{\mathcal{F}_{2}}\right)(\xi, \eta)-\left(c_{1} e^{\mathcal{F}_{1}}-c_{2} e^{\mathcal{F}_{2}}\right)(\xi, \eta)+\left(c_{1} e^{\mathcal{F}_{1}}-c_{2} e^{\mathcal{F}_{2}}\right)(\xi, a)=0 \\
& (\eta-a) c_{1} \frac{\partial}{\partial \eta} e^{\mathcal{F}_{1}(\xi, \eta)}-(\eta-a) c_{2} \frac{\partial}{\partial \eta} e^{\mathcal{F}_{2}(\xi, \eta)}-c_{1} e^{\mathcal{F}_{1}(\xi, \eta)}+c_{2} e^{\mathcal{F}_{2}(\xi, \eta)} \\
& +c_{1} e^{\mathcal{F}_{1}(\xi, a)}-c_{2} e^{\mathcal{F}_{2}(\xi, a)}=0 \\
& c_{1}\left\{(\eta-a) \frac{\partial}{\partial \eta} e^{\mathcal{F}_{1}(\xi, \eta)}-e^{\mathcal{F}_{1}(\xi, \eta)}+e^{\mathcal{F}_{1}(\xi, a)}\right\}-c_{2}\left\{(\eta-a) \frac{\partial}{\partial \eta} e^{\mathcal{F}_{2}(\xi, \eta)}\right. \\
& \left.+e^{\mathcal{F}_{2}(\xi, \eta)}-e^{\mathcal{F}_{2}(\xi, a)}\right\}=0 \\
& c_{1}\left\{(\eta-a) \frac{\partial}{\partial \eta} e^{\mathcal{F}_{1}(\xi, \eta)}-e^{\mathcal{F}_{1}(\xi, \eta)}+e^{\mathcal{F}_{1}(\xi, a)}\right\}=c_{2}\left\{(\eta-a) \frac{\partial}{\partial \eta} e^{\mathcal{F}_{2}(\xi, \eta)}\right. \\
& \left.-e^{\mathcal{F}_{2}(\xi, \eta)}+e^{\mathcal{F}_{2}(\xi, a)}\right\}, \\
& \frac{c_{2}}{c_{1}}=\frac{(\eta-a) e^{\mathcal{F}_{1}(\xi, \eta)} \frac{\partial}{\partial \eta} \mathcal{F}_{1}(\xi, \eta)-e^{\mathcal{F}_{1}(\xi, \eta)}+e^{\mathcal{F}_{1}(\xi, a)}}{(\eta-a) e^{\mathcal{F}_{2}(\xi, \eta)} \frac{\partial}{\partial \eta} \mathcal{F}_{2}(\xi, \eta)-e^{\mathcal{F}_{2}(\xi, \eta)}+e^{\mathcal{F}_{2}(\xi, a)}}
\end{aligned}
$$

Similarly, one can show that

$$
\frac{c_{2}}{c_{1}}=\frac{(\xi-a) e^{\mathcal{F}_{1}(\xi, \eta)} \frac{\partial}{\partial \xi} \mathcal{F}_{1}(\xi, \eta)-e^{\mathcal{F}_{1}(\xi, \eta)}+e^{\mathcal{F}_{1}(a, \eta)}}{(\xi-a) e^{\mathcal{F}_{2}(\xi, \eta)} \frac{\partial}{\partial \xi} \mathcal{F}_{2}(\xi, \eta)-e^{\mathcal{F}_{2}(\xi, \eta)}+e^{\mathcal{F}_{2}(a, \eta)}}
$$

Putting the values of $c_{1}$ and $c_{2}$, one has the required result.

\section{CONCLUSiON}

We have defined the coordinated exponentially convex functions. Petrovićs type inequality for exponentially convex and coordinated exponentially convex functions have been derived. We also derived Lagrange type and Cauchy type mean value theorems for Petrović's type inequality for exponentially convex and coordinated exponentially convex functions. Some new special cases are discovered. We hope that the strategies of this paper will motivate the researchers working in functional analysis, information theory and statistical theory. This is a new path for research in future. 


\section{AUthors CONTRIBUtions}

All the authors worked jointly and contributed equally. They all read and approved the final manuscript.

\section{CONFLicts OF INTEREST}

All the authors declare no conflict of interest.

\section{ACKNOWLEDGMENTS}

The authors would like to thank the Rector, COMSATS university of Islamabad, Pakistan, for providing excellent research facilities. Authors are thankful to the referees for their valuable suggestions and comments.

\section{REFERENCES}

[1] M. Alomari, M. A. Latif, On Hadmard-type inequalities for h-convex functions on the co-ordinates, Int. Journal of Math. Anal. 3(33)(2009) 1645-1656.

[2] G. Alirezaei and R. Mathar, On exponentially concave functions and their impact in information theory, Information Theory and Applications Workshop (ITA?18), San Diego, California, USA, 5(2018) 265-274.

[3] T. Antczak, (p,r)-Invex sets and functions, J. Math. Anal. Appl. (263)(2001) 355-379.

[4] M. Avriel, $r$-Convex functions, Math. Program. (2)(1972) 309-323.

[5] M. U. Awan, M. A. Noor and K. I. Noor, Hermite-Hadamard inequalities for exponentially convex functions, Appl. Math. Inform. Sci. (12)(2018) 405-409.

[6] M. K. Bakula, J. Pecarić and M. Ribicić, Companion inequalities to Jensen's inequality for m-convex and $(\alpha, m)$-convex functions, J. Inequal. Pure Appl. Math. 7, No.5 (2006) 32 pp.

[7] S. N. Bernstein, Sur les fonctions absolument monotones, Acta Math. 52 (1929) 1-66.

[8] S. Butt, J. Pecarić and A. U. Rehman, Exponential convexity of Petrović and related functional, J. Inequal. Appl. 20111(2011) 16 pp.

[9] S. S Dragomir, On Hadamards inequality for convex functions on the co-ordinates in a rectangle from the plane, Taiwanese J. Math. 5, No.4(2001) 775-788.

[10] W. Iqbal, K. M. Awan, A. U. Rehman and G. Farid and, An extension of Petrović's inequality for $h-$ convex ( $h$-concave) functions in plane, Open J. Math. Sci. 3(2019) 398-403.

[11] V.G. Mihesan, A generalization of the convexity, Seminar on functional equations, approximation and convexity, Cluj-Napoca, 1993 (Romania).

[12] M. A. Noor, Some new classes of nonconvex functions, NonlinearFunct. Anal. Appl., 11, No.1(2006) 165171.

[13] M. A. Noor and K. I. Noor, On exponentially convex functions, J. Orissa Math. Soc. 38, No.1 (2019) 33-51.

[14] M. A. Noor and K. I. Noor, New classes of strongly exponentially preinvex functions, AIMS Math. 4, No.6 (2019) 1554-1568.

[15] M. A. Noor and K. I. Noor, Strongly exponentially convex functions and their properties, J. Adv. Math. Stud. 12, No.2(2019) 177-185.

[16] M. A. Noor and K. I. Noor, Some properties of exponentially preinvex functions, FACTA Universit(NIS), 34, No.5(2019) 941-955.

[17] M. A. Noor and K. I. Noor, Strongly exponentially convex functions, U.P.B. Sci. Bull., Series A, 81, No. 4(2019) 75-84.

[18] M. A. Noor, K. I. Noor and M. U. Awan, Fractional Ostrowski Inequalities for s-Godunova-Levin Functions, Int. J. Anal. Appl. 5, No.2(2014) 167-173.

[19] C. Niclulescu and L. E. Persson, Convex functions and Their Applications, Springer, New York, 2018.

[20] S. Pal, T. K. Wong, Exponentially concave functions and a new information geometry, Glasnik Matematicki (46)(2018) 1070-1113.

[21] J. E. Pecarić, On the Petrović's inequality for convex functions, Glasnik Matematicki 18, No.38 (1983) $77-$ 85 . 
[22] M. Petrović's, Sur une fontionnelle, Publ. Math. Univ. Belgrade Id (1932) 146-149.

[23] J. Pecarić and V. Culjak, Inequality of Petrović's and Giaccardi for convex function of higher order, Southeast Asian Bull. Math. 26,(o.1 (2003) 57-61.

[24] J. Pecarić and J. Peric, Improvements of the Giaccardi and the Petrović's inequality and related Stolarsky type means, An. Univ. Craiova, Ser. Mat. Inf. 39, No.1 (2012), 65-75.

[25] J. E. Pecarić, F. Proschan and Y. L. Tong, Convex functions, partial orderings and statistical applications, Academic Press, New York, 1991.

[26] A. U. Rehman, G. Farid and V. N. Mishra, Generalized convex function and associated Petrović's inequality, Int. J. Anal. Appl. 17, No. 1(2019) 122-131.

[27] A. U. Rehman, G. Farid and W. Iqbal, More about Petrović's inequality on coordinates and related results, Kragujevac J. Math. 44, No.3 (2020) 335-351.

[28] A. U. Rehman, M. Mudessir, H. T. Fazal and G. Farid, Petrović's inequality on coordinates via $m-$ convex functions and related results, Cogent Math. 3, No.1 (2016) 11 pp.

[29] S. Rashid, M. A. Noor, K. I. Noor and F. Safdar, Integral inequalities for generalized preinvex functions, Punjab Univ.j. math. 51, No. 10 (2019) 77-91. 